Probability, Networks and Algorithms

Probability, Networks and Algorithms
PNA $\begin{aligned} & \text { Reduced-load equivalence for queues with Gaussian } \\ & \text { input }\end{aligned}$

\author{
A.B. Dieker
}

Report PNA-E0416 September 2004 
CWI is the National Research Institute for Mathematics and Computer Science. It is sponsored by the Netherlands Organization for Scientific Research (NWO).

$\mathrm{CWI}$ is a founding member of ERCIM, the European Research Consortium for Informatics and Mathematics.

CWI's research has a theme-oriented structure and is grouped into four clusters. Listed below are the names of the clusters and in parentheses their acronyms.

\section{Probability, Networks and Algorithms (PNA)}

Software Engineering (SEN)

Modelling, Analysis and Simulation (MAS)

Information Systems (INS)

Copyright (C) 2004, Stichting Centrum voor Wiskunde en Informatica

P.O. Box 94079, 1090 GB Amsterdam (NL)

Kruislaan 413, 1098 SJ Amsterdam (NL)

Telephone +31205929333

Telefax +31205924199

ISSN 1386-3711 


\title{
Reduced-load equivalence for queues with Gaussian input
}

\author{
ABSTRACT \\ In this note, we consider a queue fed by a number of independent heterogeneous Gaussian \\ sources. We study under what conditions a reduced load equivalence holds, i.e., when a subset \\ of the sources becomes asymptotically dominant as the buffer size increases. For this, recent \\ results on extremes of Gaussian processes are combined with de Haan theory. We explain how \\ the results of this note relate to square root insensitivity and moderately heavy tails.
}

2000 Mathematics Subject Classification: 60G70,60G15, 60K25

Keywords and Phrases: Gaussian processes, large deviations, ruin probabilities, extremes, regular variation

Note: The research was supported by the Netherlands Organization for Scientific Research (NWO) under grant 631.000 .002 


\title{
Reduced-load equivalence for queues with Gaussian input
}

\author{
A. B. Dieker* \\ CWI \\ P.O. Box 94079 \\ 1090 GB Amsterdam, the Netherlands \\ and \\ University of Twente \\ Faculty of Mathematical Sciences \\ P.O. Box 217 \\ 7500 AE Enschede, the Netherlands
}

\begin{abstract}
In this note, we consider a queue fed by a number of independent heterogeneous Gaussian sources. We study under what conditions a reduced load equivalence holds, i.e., when a subset of the sources becomes asymptotically dominant as the buffer size increases. For this, recent results on extremes of Gaussian processes [5] are combined with de Haan theory. We explain how the results of this note relate to square root insensitivity and moderately heavy tails.
\end{abstract}

\section{Introduction}

Consider a fluid queue fed by the superposition of $M$ independent stationary Gaussian sources with mean input rate $\mu>0$. If the buffer is drained at some constant rate $d>\mu$, the steady-state probability that the buffer content exceeds $u$ is given by

$$
P\left(\sup _{t \geq 0} Y_{t}-(d-\mu) t>u\right)
$$

where $Y$ is a centered separable Gaussian process with stationary increments. Recently, the asymptotics of this probability for $u \rightarrow \infty$ have been found under some regularity conditions $[5]$.

The probability (1) plays not only a role in queueing theory. It is well-known that it can be interpreted alternatively as the ruin probability of an insurance company with total premium rate $d$, initial capital $u$, and cumulative claim process $Y_{t}+\mu t$. The case that $Y$ is a superposition of two independent processes has recently attracted attention in the insurance literature under the name 'perturbed risk models'. We mention in particular Yang and Zhang [14], who study the influence of a Gaussian pertubation (Brownian motion) on the classical Cramér-Lundberg process and the Gamma process. See $[7,13]$ for further examples and references.

In this note, we focus on Gaussian processes, and consider a slightly more general setting than (1) by replacing the 'drift' $(d-\mu) t$ by $c t^{\beta}$ for some $\beta, c>0$. We study the question in

\footnotetext{
${ }^{*}$ The research was supported by the Netherlands Organization for Scientific Research (NWO) under grant 631.000 .002 .
} 
what cases only a subset of the $M$ input sources contribute to a high value of $\sup _{t>0} Y_{t}-c t^{\beta}$, i.e., for $u \rightarrow \infty$,

$$
P\left(\sup _{t \geq 0} \sum_{i=1}^{M} Y_{i}(t)-c t^{\beta}>u\right) \sim P\left(\sup _{t \geq 0} \sum_{i \in S} Y_{i}(t)-c t^{\beta}>u\right)
$$

where $S \subseteq\{1, \ldots, M\}$; we use the notation $f(u) \sim g(u)$ as $u \rightarrow \infty$ for $\lim _{u \rightarrow \infty} f(u) / g(u)=1$. We present necessary and sufficient conditions for $(2)$, and we say that we have reduced-load equivalence when (2) holds for some $S \neq\{1, \ldots, M\}$.

To explain the term 'reduced-load equivalence', consider two Gaussian sources with mean input rates $\mu_{1}$ and $\mu_{2}$, and suppose the buffer is drained at rate $d>\mu_{1}+\mu_{2}$. In the interval $[0, t]$, the input to the system is $Y_{1}(t)+\mu_{1} t+Y_{2}(t)+\mu_{2} t$ and the output is $d t$. Hence, if (2) holds for $c=d-\mu_{1}-\mu_{2}, \beta=1$, and $S=\{1\}$, the system behaves asymptotically as if only the first source is present and the buffer is drained at rate $d-\mu_{2}$. It is easy to see that the new load $\mu_{1} /\left(d-\mu_{2}\right)$ is smaller than the old load $\left(\mu_{1}+\mu_{2}\right) / d$. The same reasoning applies if one has more than two sources.

Our main assumptions are the following. First, the variance function $\sigma^{2}$ of $Y$ is supposed to be regularly varying at infinity with index $2 H \in(0,2)$. Moreover, we must impose that $\beta \in(H, 2 H)$; otherwise (2) cannot hold, since the key ingredient from [5], Proposition 1, cannot be applied.

The motivation for pursuing our analysis stems from recent results on certain nonGaussian queueing models $[1,8,11]$. An interesting special case of the results in [11] is $M=2$ and $Y_{1}, Y_{2}$ being on-off sources with exponentially distributed on and off periods, except for $Y_{2}$; the latter process is supposed to have activity periods with a general distribution $G$. It is then known that if the tail $P(G>x)$ of $G$ is heavier than $\exp \left(-x^{-1 / 2}\right)$, the queue length behaves asymptotically as if the second source produces traffic at a constant rate equal to its load. This does not hold if the tail of $G$ is lighter than $\exp \left(-x^{-1 / 2}\right)$.

In our opinion, it is instructive to study the Gaussian case in detail for two reasons. First, there is a vast body of literature on Gaussian processes, which makes this case relatively easy to study. Moreover, one can use the Gaussian case to 'predict' reduced-load type behavior in other models. For instance, the critical exponent $1 / 2$ also arises in the Gaussian framework, and a simple formula shows how this quantity changes if the underlying source characteristics are altered, cf. Theorem 1.

Our results are closely related to those of Zwart, Borst, and Dębicki [15]. While we present a condition that is both necessary and sufficient, it is not clear that the sufficient condition for reduced-load equivalence of [15] coincides with their necessary condition. Another difference is that only the superposition of two Gaussian sources $(M=2)$ is studied in [15]. In conclusion, we prove a condition that is better (or equally good) than the conditions of [15], but our condition is extremely simple and easy to check.

Another related paper is Hüsler and Schmid [6]. They establish exact asymptotics of (1) if $Y$ is a sum of independent Gaussian processes. The difference with the present note is that we assume stationary increments and allow general variance functions, while Hüsler and Schmid restrict the variance function to be of the form $\sum_{i} \alpha_{i} t^{2 H_{i}}$ but do not require stationarity of the increments. Another, more fundamental, difference is the type of question we ask. Exact asymptotics for (1) have already been established in [5] in the present setting, but here we focus on the reduced load equivalence (2).

The results in this note are readily adapted if $c t^{\beta}$ is replaced by a regularly varying function $\phi$; the only reason for considering $c t^{\beta}$ is to avoid cumbersome notation, cf. assumptions M1-M4 in [5]. The case $\beta \neq 1$ may also be relevant in a queueing context; see [12].

Some words for the organization of this note. Section 2 describes the results, and Section 3 gives some examples. The required proofs are contained in Section 4. 


\section{Description of the result}

We first introduce some notation. Let $Y_{1}, \ldots, Y_{M}$ be independent centered Gaussian processes with stationary increments. The finite dimensional distributions are then completely determined by their respective variance functions $\sigma_{1}^{2}, \ldots, \sigma_{M}^{2}$ : for $s, t \geq 0$,

$$
\operatorname{Cov}\left(Y_{i}(s), Y_{i}(t)\right)=\frac{1}{2}\left[\sigma_{i}^{2}(s)+\sigma_{i}^{2}(t)-\sigma_{i}^{2}(|t-s|)\right] .
$$

The sum of these processes is denoted by $Y$, so that $Y$ has stationary increments and variance function $\sigma^{2}=\sum_{i=1}^{M} \sigma_{i}^{2}$.

Given a subset $S$ of $\{1, \ldots, M\}$, we set

$$
\sigma_{S}^{2}(t):=\sum_{i \in S} \sigma_{i}^{2}(t)
$$

and $S^{c}:=\{1, \ldots, M\} \backslash S$.

Now we formulate the assumptions on the variance functions.

S1 For $i=1, \ldots, M, \sigma_{i}^{2}$ is continuous and regularly varying at infinity with index $2 H_{i}$ for some $H_{i} \in(0,1)$,

S2 For $i=1, \ldots, M, \sigma_{i}^{2}$ is ultimately continuously differentiable and its first derivative $\dot{\sigma}_{i}^{2}$ is ultimately monotone,

S3 For $i=1, \ldots, M, \sigma_{i}^{2}$ is ultimately twice continuously differentiable and its second derivative $\ddot{\sigma}_{i}^{2}$ is ultimately monotone,

S4 $\sigma(t) \leq C t^{\gamma}$ on a neighborhood of zero for some $C, \gamma>0$.

Note that $\mathbf{S 1}$ implies that the variance function $\sigma^{2}$ of the sum $Y$ is regularly varying with index $2 H$, with $H:=\max _{i=1}^{M} H_{i}$. Define

$$
S_{*}:=\left\{i \in\{1, \ldots, M\}: \sigma_{i}^{2} \text { is regularly varying with index } 2 H\right\},
$$

as the family of indices with maximum index of variation.

We start with a simple consequence of Proposition 2 of [5], which we state without proof. Note that for $\beta=1$ the condition $\beta \in(H, 2 H)$ is equivalent to $H \in(1 / 2,1)$.

Proposition 1 Let the $\sigma_{i}^{2}$ and $\sigma^{2}$ satisfy $\mathbf{S 1 - S 4}$. For $H \in(0,1)$ and $\beta \in(H, 2 H)$, we have for any $S \supseteq S_{*}$,

$$
\frac{P\left(\sup _{t \geq 0} Y(t)-c t^{\beta}>u\right)}{\sup _{t \geq 0} P\left(Y(t)-c t^{\beta}>u\right)} \sim \frac{P\left(\sup _{t \geq 0} \sum_{i \in S} Y_{i}(t)-c t^{\beta}>u\right)}{\sup _{t \geq 0} P\left(\sum_{i \in S} Y_{i}(t)-c t^{\beta}>u\right)} .
$$

Proposition 1 shows that (2) holds if and only if

$$
\sup _{t \geq 0} P\left(\sum_{i=1}^{M} Y_{i}(t)-c t^{\beta}>u\right) \sim \sup _{t \geq 0} P\left(\sum_{i \in S} Y_{i}(t)-c t^{\beta}>u\right) .
$$

The following theorem, which is the main result of this note, gives a simple necessary and sufficient condition for this to hold. It is proven in Section 4. 
Theorem 1 Let $S \supseteq S_{*}$. Under the conditions of Proposition 1, the reduced-load equivalence (2) holds if and only if

$$
\lim _{u \rightarrow \infty} \frac{u \sigma_{S^{c}}\left(u^{1 / \beta}\right)}{\sigma_{S}^{2}\left(u^{1 / \beta}\right)}=0
$$

To understand this result intuitively, it is important to gain insight into the optimizers on both sides of (4). As for the left hand side, we have

$$
\underset{t \geq 0}{\arg \sup } P\left(\sum_{i=1}^{M} Y_{i}(t)-c t^{\beta}>u\right)=\underset{t \geq 0}{\arg \inf } \frac{u+c t^{\beta}}{\sigma(t)}=\left(u \underset{t \geq 0}{\arg \inf } \frac{\sigma\left(u^{1 / \beta}\right)(1+c t)}{\sigma\left([u t]^{1 / \beta}\right)}\right)^{1 / \beta},
$$

and by the regular variation of $\sigma$, it is plausible that the optimizer in the latter expression tends to $t^{*}:=\arg \inf _{t \geq 0}(1+c t) / t^{H / \beta}$. The same reasoning applies to the right hand side. Hence, suppose that both suprema in (4) be attained for $t_{u}^{*}=\left(u t^{*}\right)^{1 / \beta}$, where $t^{*}>0$ is fixed. We then have a reduced load equivalence if and only if

$$
\Psi\left(\frac{u\left(1+c t^{*}\right)}{\sigma\left(\left(u t^{*}\right)^{1 / \beta}\right)}\right) \sim \Psi\left(\frac{u\left(1+c t^{*}\right)}{\sigma_{S}\left(\left(u t^{*}\right)^{1 / \beta}\right)}\right)
$$

or,

$$
\lim _{u \rightarrow \infty}\left[\frac{u^{2}\left(1+c t^{*}\right)^{2}}{\sigma_{S}^{2}\left(\left(u t^{*}\right)^{1 / \beta}\right)}-\frac{u^{2}\left(1+c t^{*}\right)^{2}}{\sigma^{2}\left(\left(u t^{*}\right)^{1 / \beta}\right)}\right]=\lim _{u \rightarrow \infty} \frac{u^{2}\left(1+c t^{*}\right)^{2}}{\sigma_{S}^{2}\left(\left(u t^{*}\right)^{1 / \beta}\right)} \frac{\sigma_{S^{c}}^{2}\left(\left(u t^{*}\right)^{1 / \beta}\right)}{\sigma^{2}\left(\left(u t^{*}\right)^{1 / \beta}\right)}=0 .
$$

This condition is readily seen to be equivalent to (5).

\section{Examples}

In this section, we present two examples to illustrate Theorem 1. While the first deals with two sources, the second shows that one may need a strictly larger set than $S_{*}$ for reduced-load equivalence to hold. In both examples we set $\beta=1$.

\subsection{An example with $M=2$}

The first example is related to Corollary 3.1 of [15]. Consider the sum of two processes, i.e., $M=2$. Suppose that the variance functions satisfy $\sigma_{1}^{2}(u) \sim C_{1} u^{2 H_{1}}$ and $\sigma_{2}^{2}(u) \sim C_{2} u^{2 H_{2}}$ for some constants $C_{1}, C_{2}>0$ and $H_{1}>H_{2}$. Also suppose that $H_{1}>1 / 2$. For instance, the first process is a fractional Brownian motion with long-range dependent characteristics, and the second process a standard Brownian motion or a short-range dependent Gaussian integrated process. Corollary 1 implies that a reduced-load equivalence holds if and only if $2 H_{1}>1+H_{2}$.

Of special interest is the case $H_{2}=1 / 2$, in which the condition reduces to $H_{1}>3 / 4$. Since the tail of the probability on the right hand side of $(2)$ can be written as $\exp \left(-\ell(u) u^{2-2 H_{1}}\right)$ for some slowly varying function $\ell$, a reduced-load equivalence then holds if and only if the exponent in this expression is smaller than $1 / 2$. This is the connection with the discussion in the introduction on a model with on-off sources. It illustrates that square root insensitivity or moderately heavy tails play an important role in situations with both short range dependent processes on the one hand and long range dependent processes or subexponential variables on the other hand; see, e.g., $[1,2,10,9,11]$.

It is interesting to note that the condition $H_{1}>3 / 4$ also plays a role in a different problem connected to the superposition of two independent processes. Indeed, set $\sigma_{1}^{2}(u):=u$ and $\sigma_{2}^{2}(u):=u^{2 H_{2}}$ for some $H_{2}>1 / 2$; the distribution in $C[0, T]$ of $Y_{1}+Y_{2}$ is absolutely continuous to the distribution of $Y_{1}$ (and vice versa) if and only if $H_{1}>3 / 4$; otherwise 
they are singular. These assertions are due to Cheridito [4]. However, we believe that the appearance of $3 / 4$ in both problems is rather coincidence than caused by some underlying principle.

\subsection{The set $S_{*}$ is not always the dominant set}

We now illustrate the fact that one may need a larger set than $S_{*}$ for RLE to hold. For simplicity, set $M=3$ and suppose that $Y_{1}$ is a fractional Brownian motion $B_{H_{1}}$ with Hurst parameter $H_{1}, Y_{2}$ a fractional Brownian motion $B_{H_{2}}$ with Hurst parameter $H_{2}$, and $Y_{3}$ a standard Brownian motion $B$ (all mutually independent). Let $H_{1}$ and $H_{2}$ satisfy

$$
\frac{3}{4}<H_{2}<H_{1}<\frac{H_{2}+1}{2} .
$$

It is easy to verify that Theorem 1 implies that the following does not hold:

$$
P\left(\sup _{t \geq 0} B_{H_{1}}(t)+B_{H_{2}}(t)+B(t)-t>u\right) \sim P\left(\sup _{t \geq 0} B_{H_{1}}(t)-t>u\right) .
$$

Hence, $B_{H_{1}}$ alone does not determine the asymptotics, although $S_{*}=\{1\}$. However, Theorem 1 also shows that the following does hold:

$$
P\left(\sup _{t \geq 0} B_{H_{1}}(t)+B_{H_{2}}(t)+B(t)-t>u\right) \sim P\left(\sup _{t \geq 0} B_{H_{1}}(t)+B_{H_{2}}(t)-t>u\right),
$$

i.e., the first two processes are asymptotically dominant.

\section{Proof of Theorem 1}

The proof consists of three steps. We first present an 'intermediate' necessary and sufficient condition, which is not so explicit. In the second step, this condition is shown to be necessary for (5), and the last step shows sufficiency.

For notational convenience, we set $\mu(t):=t^{1 / \beta} / c$. Moreover, we let $t^{*}$ denote the argument of the infimum of $(1+t)^{2} / t^{2 H / \beta}$ over $\mathbb{R}_{+}$, i.e., $t^{*}=H /(\beta-H)$.

\section{Step 1: auxiliary necessary and sufficient condition}

Proposition 1 implies that (2) holds if and only if

$$
\lim _{u \rightarrow \infty}\left[\inf _{t \geq 0} \frac{u^{2}(1+t)^{2}}{\sigma_{S}^{2}(\mu(u t))}-\inf _{t \geq 0} \frac{u^{2}(1+t)^{2}}{\sigma^{2}(\mu(u t))}\right] \leq 0 .
$$

Let $\epsilon>0$ be small. In the first step of the proof, we show that the condition in the preceding display is equivalent to

$$
\lim _{u \rightarrow \infty}\left[\inf _{t \in\left[t^{*} \pm \epsilon\right]} \frac{u^{2}(1+t)^{2}}{\sigma_{S}^{2}(\mu(u t))}-\inf _{t \in\left[t^{*} \pm \epsilon\right]} \frac{u^{2}(1+t)^{2}}{\sigma^{2}(\mu(u t))}\right] \leq 0
$$

where we denoted the interval $\left[t^{*}-\epsilon, t^{*}+\epsilon\right]$ by $\left[t^{*} \pm \epsilon\right]$. We only show this for the second infimum; a similar reasoning applies to the first.

Select some (large) $T>0$ such that

$$
\frac{1}{4} T^{1-H / \beta} \geq \frac{1+t^{*}}{\left(t^{*}\right)^{H / \beta}}
$$


We start by showing that the suprema in (6) can be taken over the interval $[0, T]$. To see this, note that for large $u$

$$
\inf _{t \geq T} \frac{1+t}{\sigma(\mu(u t))} \geq \inf _{t \geq T} \frac{t}{\sigma(\mu(u t))} \geq \frac{1}{2} \frac{T^{1-H / \beta}}{\sigma(\mu(u))} \geq 2 \frac{1+t^{*}}{\sigma(\mu(u))\left(t^{*}\right)^{H / \beta}} \geq \frac{1+t^{*}}{\sigma\left(\mu\left(u t^{*}\right)\right)}
$$

where the second inequality is a consequence of Theorem 1.5.3 of Bingham, Goldie and Teugels [3] (indeed, the map $t \mapsto t / \sigma(\mu(t))$ is locally bounded on $[1, \infty)$ and regularly varying with index $1-H / \beta>0)$. The last inequality follows from the definition of regular variation.

Now we show that infima cannot be attained on $[0, T] \backslash\left[t^{*} \pm \epsilon\right]$ for large $u$. Choose some $\eta>0$ such that

$$
(1+\eta)\left[\sup _{t \in[0, T] \backslash\left[t^{*} \pm \epsilon\right]} \frac{t^{H / \beta}}{1+t}+\eta\right] \leq \frac{\left(t^{*}\right)^{H / \beta}}{1+t^{*}},
$$

which is possible since $\epsilon>0$. Again exploiting the local boundedness of $\sigma^{2}$, by Theorem 1.5.2 of [3] we have for large $u$,

$$
\begin{aligned}
\inf _{t \in[0, T] \backslash\left[t^{*} \pm \epsilon\right]} \frac{1+t}{\sigma(\mu(u t))} & \geq \inf _{t \in[0, T] \backslash\left[t^{*} \pm \epsilon\right]} \frac{1+t}{\sigma(\mu(u))\left[t^{H / \beta}+\eta\right]} \\
& \geq \frac{1}{\sigma(\mu(u))\left[\sup _{t \in[0, T] \backslash\left[t^{*} \pm \epsilon\right]} \frac{t^{H / \beta}}{1+t}+\eta\right]} \\
& \geq(1+\eta) \frac{1+t^{*}}{\sigma(\mu(u))\left(t^{*}\right)^{H / \beta}},
\end{aligned}
$$

which majorizes $\inf _{t \geq 0}(1+t) / \sigma(\mu(u t))$ again by Theorem 1.5.2 of [3].

\section{Step 2: (5) implies (7)}

Before proving that (7) is equivalent to (5) by combining Step 2 and 3, we first make an observation concerning the second-order behavior of the function $\sigma_{S}^{2}(\mu(\cdot)) / \sigma^{2}(\mu(\cdot))$.

Observe that $\sigma^{2}=\sigma_{S^{c}}^{2}+\sigma_{S}^{2}$ and that $\sigma_{S^{c}}^{2}(u) / \sigma_{S}^{2}(u) \rightarrow 0$ since $S \supseteq S_{*}$, so that the function $\sigma_{S}^{2}(\mu(\cdot)) / \sigma^{2}(\mu(\cdot))$ lies in the de Haan class of $\sigma_{S^{c}}^{2}(\mu(\cdot)) / \sigma_{S}^{2}(\mu(\cdot))$ with index $2(H-$ $\left.\max _{i \in S^{c}} H_{i}\right) / \beta$, i.e., for $t>0$,

$$
\frac{\sigma_{S}^{2}(\mu(u t))}{\sigma^{2}(\mu(u t))}-\frac{\sigma_{S}^{2}(\mu(u))}{\sigma^{2}(\mu(u))} \sim\left(1-t^{2\left(\max _{i \in S^{c}} H_{i}-H\right) / \beta}\right) \frac{\sigma_{S^{c}}^{2}(\mu(u))}{\sigma_{S}^{2}(\mu(u))} .
$$

For background on de Haan theory, we refer to Chapter 3 of [3].

Now we prove the sufficiency of (5). By (8), we have

$$
\begin{aligned}
& \inf _{t \in\left[t^{*} \pm \epsilon\right]} \frac{u^{2}(1+t)^{2}}{\sigma^{2}(\mu(u t))} \\
= & \inf _{t \in\left[t^{*} \pm \epsilon\right]} \frac{u^{2}(1+t)^{2}}{\sigma_{S}^{2}(\mu(u t))}\left[\frac{\sigma_{S}^{2}(\mu(u))}{\sigma^{2}(\mu(u))}+(1+o(1))\left(1-t^{2\left(\max _{i \in S^{c}} H_{i}-H\right) / \beta}\right) \frac{\sigma_{S^{c}}^{2}(\mu(u))}{\sigma_{S}^{2}(\mu(u))}\right] \\
\geq & \frac{\sigma_{S}^{2}(\mu(u))}{\sigma^{2}(\mu(u))} \inf _{t \in\left[t^{*} \pm \epsilon\right]} \frac{u^{2}(1+t)^{2}}{\sigma_{S}^{2}(\mu(u t))} \\
+ & \frac{\sigma_{S^{c}}^{2}(\mu(u))}{\sigma_{S}^{2}(\mu(u))} \inf _{t \in\left[t^{*} \pm \epsilon\right]} \frac{u^{2}(1+t)^{2}}{\sigma_{S}^{2}(\mu(u t))}\left(1-t^{2\left(\max _{i \in S^{c}} H_{i}-H\right) / \beta}\right)(1+o(1)) \\
=: & I(u)+I I(u),
\end{aligned}
$$

and $o(1)$ is uniform in $t \in\left[t^{*} \pm \epsilon\right]$ by Theorem 3.1.16 of [3]. 
Observe that

$$
\begin{aligned}
\inf _{t \in\left[t^{*} \pm \epsilon\right]} \frac{u^{2}(1+t)^{2}}{\sigma_{S}^{2}(\mu(u t))}-I(u) & =\left(1-\frac{\sigma_{S}^{2}(\mu(u))}{\sigma^{2}(\mu(u))}\right) \inf _{t \in\left[t^{*} \pm \epsilon\right]} \frac{u^{2}(1+t)^{2}}{\sigma_{S}^{2}(\mu(u t))} \\
& =\frac{\sigma_{S^{c}}^{2}(\mu(u))}{\sigma^{2}(\mu(u))} \inf _{t \in\left[t^{*} \pm \epsilon\right]} \frac{u^{2}(1+t)^{2}}{\sigma_{S}^{2}(\mu(u t))} \\
& =\frac{u^{2} \sigma_{S^{c}}^{2}(\mu(u))}{\sigma_{S}^{4}(\mu(u))} \frac{\sigma_{S}^{2}(\mu(u))}{\sigma^{2}(\mu(u))} \inf _{t \in\left[t^{*} \pm \epsilon\right]} \frac{\sigma_{S}^{2}(\mu(u))(1+t)^{2}}{\sigma_{S}^{2}(\mu(u t))} .
\end{aligned}
$$

The infimum tends to a constant in $(0, \infty)$ by Theorem 1.5.2 of [3], and the other terms tend to zero as a consequence of (5). Step 2 is complete once it has been shown that $I I(u)$ tends to zero, or equivalently that

$$
\lim _{u \rightarrow \infty} \frac{\sigma_{S^{c}}^{2}(\mu(u))}{\sigma_{S}^{2}(\mu(u))} \inf _{t \in\left[t^{*} \pm \epsilon\right]} \frac{u^{2}(1+t)^{2}}{\sigma_{S}^{2}(\mu(u t))}\left(1-t^{2\left(\max _{i \in S^{c}} H_{i}-H\right) / \beta}\right)=0 .
$$

For this, note that the left hand side equals

$$
\begin{aligned}
& \lim _{u \rightarrow \infty} \frac{u^{2} \sigma_{S^{c}}^{2}(\mu(u))}{\sigma_{S}^{4}(\mu(u))} \inf _{t \in\left[t^{*} \pm \epsilon\right]} \frac{\sigma_{S}^{2}(\mu(u))(1+t)^{2}}{\sigma_{S}^{2}(\mu(u t))}\left(1-t^{2\left(\max _{i \in S^{c}} H_{i}-H\right) / \beta}\right) \\
= & \lim _{u \rightarrow \infty} \frac{u^{2} \sigma_{S^{c}}^{2}(\mu(u))}{\sigma_{S}^{4}(\mu(u))} \inf _{t \in\left[t^{*} \pm \epsilon\right]} \frac{(1+t)^{2}}{t^{2 H / \beta}}\left(1-t^{2\left(\max _{i \in S^{c}} H_{i}-H\right) / \beta}\right),
\end{aligned}
$$

by Theorem 1.5.2 of [3] and the fact that $1-t^{2\left(\max _{i \in S^{c}} H_{i}-H\right) / \beta}$ is bounded away from $\pm \infty$ on $\left[t^{*} \pm \epsilon\right]$. Evidently, (5) implies that the limits in the preceding display are equal to zero.

\section{Step 3: (7) implies (5)}

Now suppose that (5) does not hold. Observe that

$$
1-t^{2\left(\max _{i \in S^{c}} H_{i}-H\right) / \beta} \leq 1-\frac{1}{2}\left(t^{*}\right)^{2\left(\max _{i \in S^{c}} H_{i}-H\right) / \beta}=: \alpha<1
$$

for $t \in\left[t^{*} \pm \epsilon\right]$ for $\epsilon>0$ small enough. Hence, for large $u$,

$$
\inf _{t \in\left[t^{*} \pm \epsilon\right]} \frac{u^{2}(1+t)^{2}}{\sigma^{2}(\mu(u t))} \leq \inf _{t \in\left[t^{*} \pm \epsilon\right]} \frac{u^{2}(1+t)^{2}}{\sigma_{S}^{2}(\mu(u t))}\left[\frac{\sigma_{S}^{2}(\mu(u))}{\sigma^{2}(\mu(u))}+(\alpha+o(1)) \frac{\sigma_{S^{c}}^{2}(\mu(u))}{\sigma_{S}^{2}(\mu(u))}\right],
$$

so that for large $u$

$$
\begin{aligned}
& \inf _{t \in\left[t^{*} \pm \epsilon\right]} \frac{u^{2}(1+t)^{2}}{\sigma_{S}^{2}(\mu(u t))}-\inf _{t \in\left[t^{*} \pm \epsilon\right]} \frac{u^{2}(1+t)^{2}}{\sigma^{2}(\mu(u t))} \\
\geq & \frac{u^{2} \sigma_{S^{c}}^{2}(\mu(u))}{\sigma_{S}^{4}(\mu(u))} \inf _{t \in\left[t^{*} \pm \epsilon\right]} \frac{\sigma_{S}^{2}(\mu(u))(1+t)^{2}}{\sigma_{S}^{2}(\mu(u t))}\left[\frac{\sigma_{S}^{2}(\mu(u))}{\sigma^{2}(\mu(u))}-\alpha+o(1)\right] \\
\geq & \frac{1-c}{2} \frac{u^{2} \sigma_{S^{c}}^{2}(\mu(u))}{\sigma_{S}^{4}(\mu(u))},
\end{aligned}
$$

which does not converge to zero.

\section{Acknowledgment}

The author is grateful to M.A. Lifshits and M. Mandjes for valuable comments. 


\section{References}

[1] R. Agrawal, A. Makowski, and Ph. Nain. On a reduced load equivalence for fluid queues under subexponentiality. Queueing Systems Theory Appl., 33:5-41, 1999.

[2] S. Asmussen, C. Klüppelberg, and K. Sigman. Sampling at subexponential times, with queueing applications. Stochastic Process. Appl., 79:265-286, 1999.

[3] N. H. Bingham, C. M. Goldie, and J. L. Teugels. Regular variation. Cambridge University Press, Cambridge, 1989.

[4] P. Cheridito. Mixed fractional Brownian motion. Bernoulli, 7:913-934, 2001.

[5] A. B. Dieker. Extremes of Gaussian processes over an infinite horizon. To appear in Stochastic Process. Appl., 2004.

[6] J. Hüsler and Ch. M. Schmid. Extreme values of a portfolio of Gaussian processes and a trend. Preprint, 2004.

[7] M. Huzak, M. Perman, H. Šikić, and Z. Vondraček. Ruin probabilities and decompositions for general perturbed risk processes. Preprint, 2003.

[8] P. Jelenković and A. Lazar. Asymptotic results for multiplexing subexponential on-off processes. Adv. in Appl. Probab., 31:394-421, 1999.

[9] P. Jelenković and P. Momčilović. Large deviation analysis of subexponential waiting times in a processor-sharing queue. Math. Oper. Res., 28:587-608, 2003.

[10] P. Jelenković and P. Momčilović. Large deviations of square root insensitive random sums. Math. Oper. Res., 29:398-406, 2004.

[11] P. Jelenković, P. Momčilović, and B. Zwart. Reduced load equivalence under subexponentiality. Queueing Syst., 46:97-112, 2004.

[12] T. Konstantopoulos and V. Anantharam. Optimal flow control schemes that regulate the burstiness of traffic. IEEE/ACM Transactions on Networking, 3:423-432, 1995.

[13] H. Schmidli. Distribution of the first ladder height of a stationary risk process perturbed by $\alpha$-stable Lévy motion. Insurance Math. Econom., 28:13-20, 2001.

[14] H. Yang and L. Zhang. Spectrally negative Lévy processes with applications in risk theory. Adv. in Appl. Probab., 33:281-291, 2001.

[15] B. Zwart, S. Borst, and K. Dȩbicki. Reduced-load equivalence for Gaussian processes. To appear in Oper. Res. Lett., 2004. 